\title{
Optics with complex materials and (sub)nanostructures: introduction
}

\author{
Akhlesh Lakhtakia, ${ }^{1,2, *}$ (i) Reuven Gordon, ${ }^{3,4}$ (i) Tom G. Mackay, ${ }^{1,5}$ (i) and Olivier J. F. Martin ${ }^{6}$ \\ ${ }^{1}$ NanoMM-Nanoengineered Metamaterials Group, Department of Engineering Science and Mechanics, The Pennsylvania State University, \\ University Park, Pennsylvania 16802, USA \\ ${ }^{2}$ Material Architecture Center and Department of Electronics Engineering, Indian Institute of Technology (BHU), Varanasi 221005, \\ Uttar Pradesh, India \\ ${ }^{3}$ Center for Advanced Materials and Related Technologies (CAMTEC), University of Victoria, 3800 Finnerty Road, Victoria, \\ British Columbia V8P 5C2, Canada \\ ${ }^{4}$ Department of Electrical and Computer Engineering, University of Victoria, 3800 Finnerty Road, Victoria, British Columbia V8P 5C2, Canada \\ ${ }^{5}$ School of Mathematics and Maxwell Institute for Mathematical Sciences, University of Edinburgh, Edinburgh EH9 3FD, UK \\ ${ }^{6}$ Nanophotonics and Metrology Laboratory, Swiss Federal Institute of Technology Lausanne, Lausanne CH-1015, Switzerland \\ *Corresponding author: akhlesh@psu.edu
}

Received 29 July 2019; posted 29 July 2019 (Doc. ID 374173); published 1 August 2019

Comprising 15 contributed papers and three invited review papers, this feature issue is focused on theoretical and experimental research on electromagnetic optics with complex materials and (sub)nanostructures. (๑2019 Optical Society of America

https://doi.org/10.1364/JOSAB.36.000CM1

Scientific and technological progress for the last two decades has been dominated by the conceptualization, characterization, fabrication, and application of many different classes of materials and structures. Although some are found in nature, others are entirely synthetic, created by chemical and physical processes, often at the nanoscale. Nanostructural engineering is often used to fabricate materials and structures with the same chemical composition but different optical response characteristics depending on nanoscale morphology. Certain materials are multi-phase nanocomposites designed for desirable response characteristics that are otherwise unavailable.

This feature issue is focused on theoretical and experimental research on electromagnetic optics with complex materials and nanostructures. Complex materials cannot be macroscopically described locally by just a single frequency-domain local constitutive parameter that is either a permittivity or a permeability. Complex composite materials arise from combining simple materials. By judicious design, their response characteristics may exceed, or be entirely different from, those of their component materials. Material complexity may engender multifunctionality. Interaction of electromagnetic fields with (sub)nanostructures requires quantum and/or semiclassical approaches for understanding, analysis, and design.

Original unpublished contributions were solicited on the following topics for publication after review: chiral, anisotropic, and bianisotropic materials; topological materials; low-dimensional materials; nonlinear and/or nonlocal materials; materials with (sub)nanoscale morphology including nanocomposite materials; quantum-well structures; macroscopic, mesoscopic, and atomic-scale models; and optical and optoelectronic applications. A few key researchers were invited to write review papers.

We received a total of 29 submissions, of which 18 were accepted for publication. Three of the accepted papers are invited review papers on: active metamaterial absorbers, polarimetry of biansiotropic materials, and optics of hyperbolic materials. The 15 contributed papers span: harmonic generation, metasurfaces, nanophotonics, nanoplasmonics, scattering and radiation, sensing, and surface waves. We expect that all 18 shall be of service to the optics and photonics research community. 